\title{
Antidiabetikum mit Herzschutzfaktor?
}

- Typ-2-Diabetiker sterben nicht am hohen Blutzucker, sondern an makrovaskulären Erkrankungen, die das Resultat vieler metabolischer Risikofaktoren sind. Daher macht es Sinn, eine Therapie zu wählen, die möglichst an allen Problemen ansetzt. Mit einem GLP-1-Agonisten wie Exenatide hat man hier gute Chancen. Und in naher Zukunft auch mit nur einer Injektion pro Woche.

Eine wichtige Rolle bei dem erhöhten Atheroskleroserisiko kommt dem Proinsulin zu. Darauf wies Prof. Thomas Forst aus Mainz hin. Proinsulin taucht bei zunehmender Betazellerschöpfung vermehrt im Blut auf. Laut Forst steigert es die Insulinresistenz, fördert die Hypertonie und wirkt atherogen. „Proinsulin ist ein unabhängiger kardiovaskulärer Risikofaktor!“ Umso wichtiger sei der Einsatz von Antidiabetika, die die Betazellen schonen, wie das bei Exenatide der Fall ist.
Exenatide muss bisher zweimal täglich injiziert werden. Im großen Studienprogramm DURATION wurde jetzt eine neue, einmal wöchentliche Darreichungsform erfolgreich getestet. In den DURATION-Studien war Exenatide bei $\mathrm{HbA}_{1 c^{-}}$ und Gewichtsreduzierung allen oralen Antidiabetika überlegen. Zusätzlich kam es zu einer relevanten Blutdrucksenkung. In einer Subgruppenanalyse erfuhren $\mathrm{Pa}$ tienten mit Hypertonie eine mittlere Druckreduktion von $11 \mathrm{mmHg}$.

Ob diese pleiotropen Effekte positive Auswirkungen auf die kardiovaskuläre Ereignisrate haben, soll jetzt in der Studie EXSCEL bei etwa 9500 Typ-2-Diabetikern mit der einmal wöchentlichen Darreichung von Exenatide geprüft werden.

\footnotetext{
- Sarah Pampel

Quelle: Redaktionsgespräch „Differenzialtherapie im Fokus“, München 2. November 2010
} (Veranstalter: Lilly)
Neue Mikrosphären-Technologie

Exenatide nur noch einmal wöchentlich

Für die neue Galenik ist eine Mikrosphären-Technologie entwickelt worden. Die subkutan zu injizierenden Mikrosphären bestehen aus einem Polymer, das traditionell für resorbierbares Nahtmaterial verwendet wird. Daraus wird der Wirkstoff kontinuierlich freigesetzt, wobei das Polymer vollständig zu $\mathrm{CO}_{2}$ und $\mathrm{H}_{2} \mathrm{O}$ abgebaut wird. Nach der zweiten Woche wird der Wirkspiegel, nach 10-12 Wochen Therapie ein Steady State erreicht. Mit dem neuen Präparat, das den Namen Bydureon ${ }^{\circledR}$ tragen soll, wird ein gleichmäßiges, lang anhaltendes Wirkprofil erzeugt. Die Daten belegen, dass so nächtlicher und Nüchternblutzucker besonders gut reguliert werden können. Dagegen ist die zweimal tägliche Darreichung von Exenatide postprandial besonders effektiv. Die Zulassung in Europa ist beantragt und wird für 2011 erwartet.

\section{Antientzündliche Medikation speziell für COPD-Kranke \\ Weniger Exazerbationen, bessere Lungenfunktion}

- Der PDE-4-Hemmer Roflumilast schützt COPD-Patienten infolge seines antiinflammatorischen Wirkansatzes vor Exazerbationen und verbessert die Lungenfunktion.

Da viele COPD-Patienten trotz Therapie symptomatisch bleiben und Exazerbationen erleben, besteht ein Bedarf nach Medikamenten mit neuartigen Wirkmechanismen, so Prof. Peter Barnes, London. Ein besonderes Problem sei die COPDspezifische Entzündung, die auf Steroide nicht anspricht. Die Entzündungsprozesse bleiben nicht auf die Atemwege beschränkt, sondern können auch andere Organsysteme erfassen und die typischen Komorbiditäten der COPD auslösen.

Mit dem PDE-4-Hemmer Roflumilast (Daxas ${ }^{\circledR}$ ) steht erstmals eine Therapie zur Verfügung, die sich gezielt gegen die inflammatorische Komponente der COPD richtet. Der neue Wirkstoff wurde in vier



COPD-Patient: Je mehr Exazerbationen, desto schlechter die Prognose.

randomisierten, placebokontrollierten Studien mit insgesamt knapp 5000 COPD-Patienten über sechs oder zwölf Monate untersucht.

In die beiden 12-Monats-Studien wurden rund 3000 Patienten mit schweren Atemwegsobstruktionen (postbronchodilatatorisches $\mathrm{FEV}_{1} \leq 50 \%$ ) und mindes- tens einer Exazerbation im vorausgegangenen Jahr eingeschlossen. Eine Standardtherapie mit lang wirksamen Betaagonisten (LABA) war erlaubt. Die gepoolten Daten dieser Studien zeigen laut Prof. Fernando J. Martinez, Ann Arbor/ USA, dass Roflumilast ( $500 \mu \mathrm{g} / \mathrm{d}$ ) eine statistisch signifikante Reduktion der Exazerbationen und eine Verbesserung der Lungenfunktion bewirkt. Eine deutliche Abnahme der Exazerbationen um $20,7 \%$ ( $p=0,001$ ) wurde auch beobachtet, wenn Roflumilast zusätzlich zu LABA verabreicht wurde.

Die Substanz wurde gut vertragen. Als häufigste Nebenwirkungen wurden eine leichte Nausea, Diarrhö und Gewichtsabnahme berichtet.

\footnotetext{
- Abdol A. Ameri

Quelle: Satellitensymposium „A deeper look into COPD“, ERS-Kongress, Barcelona, 19. Sep-
} tember 2010 (Veranstalter: Nycomed) 